\title{
Esencias Florales de Bach y Reflexión Personal Sobre el Aprendizaje
}

UT. Revista de Ciències de l'Educació

Desembre 2010. Pag. 7-16

ISSN 1135-1438

http://pedagogia.fcep.urv.cat/revistaut

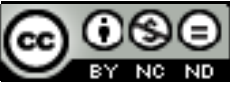

\author{
María Suz Pompa ${ }^{\mathrm{a}}$ \\ Boris C. Rodríguez Martín ${ }^{\text {b }}$ \\ Rebut: 17/11/2010 Acceptat: 01/12/2010
}

\section{Resumen}

Se realizó un estudio aleatorizado a doble ciego-placebo con el objetivo de valorar la efectividad de la metodología para el desarrollo de la reflexión personal en estudiantes de primer año de Psicología, así como la acción catalizadora de las esencias florales sobre la misma. La muestra estuvo conformada por 32 voluntarios, divididos en dos grupos de 16 estudiantes cada uno. Se pudo determinar que la metodología empleada resultó efectiva para el desarrollo de los indicadores del proceso de reflexión personal sobre el aprendizaje en estudiantes de psicología. La fórmula CHB-WAL-WOA demostró ser efectiva para el indicador autovaloración de las cualidades personales sobre la actividad de aprendizaje, e influir favorablemente en el indicador crítica objetiva acerca del conocimiento obtenido.

Palabras clave: Reflexión personal, esencias florales de Bach

\section{Bach's Flower Remedies and Personal Reflection on Learning}

\begin{abstract}
\footnotetext{
a Universidad Cental «Marta Abreu» de Las Villas - Cuba

b Universidad Cental «Marta Abreu» de Las Villas - Cuba
}

We conducted a randomized double blind-placebo with the aim of evaluating the efficacy of the methodology for the development of personal reflection in students of first year psychology as well as the catalytic action of flower essences on it. The sample consisted of 32 volunteers, divided into two groups of 16 students each. It was determined that the methodology was effective for the development of indicators of personal reflection on learning in psychology students. The formula CHB-WAL-WOA was effective for self-assessment indicator of the personal qualities of 
the learning activity, and favorably affects the indicator objective criticism on the acquired knowledge.

Key words: Personal Reflection, Bach's Flower Remedies

\section{Introducción}

En el perfil del profesional de la Psicología, se declara como propósito principal de su labor: la promoción, el mantenimiento, la restauración y la optimización del bienestar subjetivo. Al mismo tiempo, se plantea la necesidad de su inserción en programas de transformación en las diferentes dimensiones de expresión de su objeto (Individual, Grupal, Institucional y Macrosocial) y actuar como agente de cambio en las diferentes esferas de actuación profesional: Educacional y del Desarrollo, Clínica y de la Salud, Social o Laboral y de las Organizaciones. Su labor profesional como agente de cambio, también debe manifestarse en los diferentes modos de actuación, declarados como: Prevención y Promoción, Diagnóstico, Evaluación y Selección, Orientación y Asesoría, Intervención Psicológica, Investigación, Formación, Docencia y Entrenamiento. La efectividad de su actuación puede apoyarse en el desarrollo de cualidades de personalidad y mecanismos de autorregulación tales como la reflexión personal acerca de cómo tiene lugar la asimilación de sus conocimientos, habilidades y hábitos durante su preparación.

Se considera la reflexión personal como la forma en que la persona interpreta, a partir de su personalidad y de manera activa, determinados contenidos de su conciencia individual necesarios para la realización exitosa de la actividad, se dirige a la autoorganización del sujeto, a la comprensión de sí mismo, de su propio modo de hacer, en estrecha relación con su autovaloración como personalidad integral que realiza y desarrolla su individualidad; es un «mecanismo psicológico fundamental que garantiza el funcionamiento del pensamiento como sistema autorregulador». (Zajárova y Bostmánova, 1987, p. 159)

Tomando como base estos criterios, la autora principal de este trabajo (2008) ofrece la definición de reflexión personal del profesor sobre la dirección del proceso docente educativo: Mecanismo de autorregulación durante el cual el profesor somete la dirección del proceso docente educativo a una reflexión antes, durante y después de la misma, permitiéndole la modificación de sus contenidos psíquicos en la proposición y alcance de metas encaminadas a perfeccionar su quehacer profesional.

Partiendo de esta base (Suz, 2008) se ha estructurado toda una metodología para su desarrollo. Consideran los autores que esta metodología también puede ser utilizada para el desarrollo de la reflexión personal de los estudiantes sobre la actividad de estudio, concibiendo que la misma se manifiesta cuando los estudiantes someten a reflexión antes, durante y después, la actividad de aprendizaje, lo que permite la modificación de sus contenidos psíquicos en la proposición y alcance de metas encaminadas a perfeccionarla, y por ende del modo de enfrentarla. 
En este trabajo se concibe el papel de la autorregulación de la personalidad del estudiante en la ejecución del proceso de aprendizaje, con un enfoque integrador, dado por la manifestación de todos los procesos psíquicos señalados como componentes de la reflexión personal: la toma de conciencia respaldada por los aprendizajes, que da lugar a la crítica acerca de sus conocimientos, actos y circunstancias en que desarrolla su actividad, la reelaboración de métodos procedimientos y modos de actuar, como resultado de tal toma de conciencia y de la autovaloración de sus posibilidades y limitaciones para alcanzar los objetivos propuestos para el desarrollo del proceso docente educativo.

Todo lo anteriormente expresado, posibilita argumentar el carácter personológico de la reflexión personal acerca de su aprendizaje, pues durante esta el estudiante expresa plenamente sus potencialidades, aprovechando sus recursos de forma efectiva, e implicándose emocionalmente: toma conciencia de la necesidad de analizar, atribuyendo un sentido personal, el conjunto de conocimientos, ideas, juicios, experiencias, conceptos, y criterios, que fundamentan su análisis del modo en que ha ejecutado y ejecuta el proceso de aprendizaje, su valoración de alternativas para tomar decisiones sobre cómo puede realizarlo; considerándolo importante para determinar sus metas y realizar el esfuerzo volitivo necesario para avanzar hacia su propio desarrollo.

Este proceso puede ser auxiliado por herramientas catalizadoras: las esencias florales de Edward Bach. Los resultados observados en la psicoterapia (Rodríguez y Orozco, 2005 y Rodríguez, 2009), donde han sido utilizadas como catalizadoras de las acciones terapéuticas que potencian la autoconciencia, la autorregulación, la motivación y búsqueda de sentido, etc. También se han registrado experiencias sumamente interesantes con la utilización de las esencias florales para la estimulación de los procesos cognitivos básicos en niños con retraso mental ligero (Rodríguez, 2001). Estos remedios son inocuos y, al no basar su acción en los principios activos de las plantas, sino en el probable almacenamiento en el agua de un patrón de información determinado (Rivas, 2009), se encuentran carentes de efectos secundarios y reacciones adversas, por lo que pueden ser utilizados para activar las potencialidades de los estudiantes.

Si se toma en consideración que psicoterapia es aprendizaje (Kandel, 2001), puede considerarse entonces emplear las esencias florales como catalizadoras de la reflexión personal del estudiante acerca de su aprendizaje, durante el proceso pedagógico. En criterio de los autores, entre las esencias florales propuestas por Bach hallamos tres que resultan de particular importancia para ser empleadas como catalizadoras del mecanismo de reflexión personal sobre el aprendizaje. Estas son: Wild Oat (WOA), es aplicable a las personas con metas vagas o no definidas (Rodríguez y Orozco, 2005), brinda la certeza de que el camino elegido es el correcto, o no; afianza la vocación, por lo que aporta dirección y sentido al proceso de reflexión; Walnut (WAL), favorece la adaptación al cambio que la nueva etapa trae aparejado y brinda protección contra las influencias y juicios de otras personas que interfieran con los propios, y Chestnut Bud (CHB) permite el reconocimiento de los errores, y posibilita un análisis crítico de 
los mismos, sobre la base de la autovaloración, y en la valoración de alternativas para no volver a cometerlos. Está muy relacionada con la autoconciencia. (Rodríguez y Orozco, 2005)

Por todo lo anterior, constituyen objetivos de este trabajo: (1) Valorar la efectividad de la metodología para el desarrollo de la reflexión personal de los estudiantes de primer año de Psicología sobre la actividad de aprendizaje, y (2) Valorar la efectividad de la fórmula (CHB-WAL-WOA) como catalizadora de la metodología propuesta.

\section{Método}

Durante el diagnóstico realizado en las primeras semanas de incorporación de los estudiantes de primer año de Psicología del curso 2009-2010, para conocer características relacionadas con la actividad de estudio, se aplicó la técnica «¿Cómo estudias?», la cual reflejó que la mayoría de los estudiantes del año presentan dificultades en el conocimiento y utilización de métodos adecuados para estudiar, así como con la organización de este proceso, tendiendo a la reproducción memorística, sin existir una autovaloración de sus procedimientos y métodos de estudio, ni de sus características personales. Todo ello evidencia la presencia de un bajo nivel de reflexión personal de estos estudiantes en relación con la actividad de estudio.

Se realizó un estudio aleatorizado a doble ciego-placebo, para el que se seleccionó una muestra de 32 voluntarios en el grupo; que fue a su vez dividida, de forma aleatoria simple, en dos grupos de 16 sujetos: a uno se les suministró la fórmula CHBWAL-WOA y al otro se les suministró el placebo. La duración de ambos tratamientos fue de 21 días; ingiriendo 4 gotas 4 veces al día (desayuno, almuerzo, comida, y antes de irse a dormir). Los estudiantes debían registrar cada vez que tomaban las gotas, y los efectos que les producía. Se les advierte que en caso de sufrir alguna reacción adversa, de malestar, debían dejar el tratamiento de forma inmediata (cosa que no ocurrió en ningún caso). Al mismo tiempo se aplicó la metodología con el fin de desarrollar la reflexión personal sobre la actividad de aprendizaje, a la totalidad del grupo de alumnos (54). Esta metodología consiste en una variante de la empleada para el desarrollo de la reflexión personal del profesor en cuanto a la dirección del proceso docente educativo. (Suz Pompa, 2008)

La variante aplicada consta de los pasos siguientes: (1) Diagnóstico; (2) Información sobre los indicadores a tener en cuenta para el análisis; (3) Autoevaluación de la presencia de los indicadores en las actividades docentes orientadas; (4) Control sistemático de la presencia de los resultados obtenidos en las actividades docentes ejecutadas, y (5) Control sistemático de la autoevaluación para ver la presencia de los indicadores en las actividades docentes ejecutadas. La metodología fue aplicada a la totalidad de los alumnos durante 4 semanas, teniendo en cuenta que este sería el período de 21 días correspondiente a la ingestión de la fórmula de esencias florales propuesta. Durante la primera semana se realizó una actividad docente problémica. 
Al finalizar se les orienta reflejar, de modo escrito, cómo están presentes en su ejecución los indicadores que conforman el mecanismo de la reflexión personal sobre la actividad de aprendizaje. Para esto se les explica el contenido de cada uno de ellos: crítica objetiva sobre los conocimientos obtenidos (C), consiste en realizar una autovaloración acerca de si los conocimientos adquiridos son suficientes para resolver la tarea planteada; reevaluación de los propios procedimientos metodológicos empleados durante el estudio (R), consiste en realizar una autovaloración acerca de si los procedimientos empleados para adquirir los conocimientos fueron los adecuados, y suficientes o no para resolver la tarea planteada, fundamentando por qué; autovaloración de las cualidades personales y su incidencia en la obtención de los conocimientos (A), consiste en realizar una autovaloración acerca de cómo las cualidades personales contribuyeron o limitaron sus posibilidades para adquirir los conocimientos adecuados y suficientes para resolver la tarea planteada, fundamentando por qué; presencia de intenciones para la modificación de procedimientos y/o características personales para la obtención de los conocimientos (D), consiste en realizar una proyección acerca de modificaciones, ya sea en sus cualidades personales o en sus procedimientos para adquirir los conocimientos adecuados y suficientes, para resolver la tarea planteada, fundamentando por qué $y$, por último, ejecución reflexiva de la manifestación de sus conocimientos $(E)$, consiste en la realización de la tarea empleando los conocimientos adecuados y suficientes para resolver la tarea planteada, basando sus respuestas en argumentos científicos, sustentados en elaboraciones personales.

Durante las semanas segunda y la tercera se continúa sistematizando el empleo y dominio de los elementos para realizar la reflexión personal sobre la actividad de aprendizaje, aplicándoseles preguntas escritas. Luego de ser ejecutadas, se les solicita un reporte oral y otro escrito acerca de cómo se manifestaron en dicha ejecución cada uno de los indicadores. En la cuarta semana tiene lugar la prueba parcial de la asignatura y se emplean los resultados de la misma, luego de conocidos por los alumnos, para que realicen la reflexión personal sobre la actividad de aprendizaje. Esta semana coincide con el término de consumo de las gotas, ya fueran a modo de fórmula, o a modo de placebo.

Para caracterizar la presencia de la reflexión personal sobre la actividad de aprendizaje, identificada como variable dependiente de esta investigación, dado su carácter subjetivo, no puede ser medida a simple vista, resultando necesaria su operacionalización, a través de determinados indicadores que posibiliten apreciar su manifestación. Para tal efecto, se consideran los criterios teóricos y metodológicos expuestos anteriormente La definición operacional de la variable dependiente se expresa a través de los indicadores anteriormente descritos evaluados de forma individual en escala ordinal como: muy bajo; medio y alto. Tras asignar aleatoriamente los sujetos a cada grupo, y antes de iniciar los tratamientos, se procedió a evaluar el estado de los distintos indicadores de la variable a considerar durante la intervención. 
Las esencias para la realización del experimento fueron suministradas por Healing Herbs LTD y preparadas en la Farmacia Homeopática de la ciudad de Santa Clara en una dilución alcohólica al $10 \%$. El análisis de los resultados se realiza mediante el SPSS/Windows 11.0 donde, por el nivel de medición de las variables, se aplican pruebas no paramétricas: U de Mann-Whitney, comparaciones ínter-grupo, y Friedman, comparaciones intra-grupo.

\section{Resultados}

\section{Comparaciones inter-grupos}

Los resultados ilustran que antes de iniciar el experimento no existían diferencias significativas entre los indicadores, con la excepción de $A$ [U $(N 1=N 2=16)=43,5$, $\mathrm{p}<.05]$, referida a la autovaloración de los procedimientos y características personales que inciden favorable o desfavorablemente en los resultados alcanzados en el aprendizaje. Los estudiantes que conformaron el grupo placebo se encontraban muy por encima de los que conformaron el grupo fórmula para este indicador. (Gráfico 1)

Al transcurrir la primera semana de estimulación, el resultado de mayor interés en ese momento lo constituyó el valor del indicador $A[U(N 1=N 2=16)=88, p>.05]$, donde los sujetos que ingierieron la fórmula lograron emparejarse en los resultados con los que estaban tomando el placebo, lo cual habla de un efecto positivo de la fórmula en esta área, ya en el momento del diagnóstico. (Gráfico 1)

Al transcurrir 15 días, lo que más resaltó fue la ausencia de diferencias significativas entre ambos grupos. El Gráfico 1 muestra indicios de avances en el grupo placebo. Este hecho puede ser un indicador de que las acciones pedagógicas habían comenzado a surtir efecto para ambos grupos. En el último corte evaluativo se mantuvieron ambos grupos funcionando sin diferencias significativas entre las variables estudiadas. (Gráfico 1)

\section{Comparaciones intra-grupo}

Se comienza con $C$, donde se observaron cambios significativos en el grupo formula $\left[\chi^{2}(3)=25.89 ; p<.001\right]$; mientras que en el grupo placebo no ocurrieron $\left[\chi^{2}(3)=6.4\right.$; $\mathrm{p}>$.05]. El Gráfico 2 ilustra la progresión ascendente de los cambios en el grupo fórmula.

Para el caso del indicador $\mathrm{R}$, los cambios fueron significativos para ambos grupos: fórmula $\left[\chi^{2}(3)=31.22, p<.001\right]$ y placebo $\left[\chi^{2}(3)=14.21, p<.001\right]$, atribuidos en su mayor parte a las acciones pedagógicas relacionadas con la metodología para el desarrollo de la reflexión personal. Aunque pudo también apreciarse la progresión del grupo fórmula (Gráfico 2). 
Los resultados del indicador $\mathrm{A}$ no hicieron más que confirmar la efectividad de la fórmula $\left[\chi^{2}(3)=21.68, p<.001\right]$, sobre el placebo $\left[\chi^{2}(3)=5.382, p>.1\right]$, en la activación de una adecuada autovaloración de las cualidades personales (Gráfico 2).

El indicador $D$ mostró avances muy parejos en ambos grupos: fórmula $\left[\chi^{2}(3)=14.88\right.$, $p<0,005]$ y placebo $\left[\chi^{2}(3)=17.11, p<.005\right]$. Aunque en el grupo placebo estos fueron más estables (Gráfico 2). Similares resultados se observan para el indicador E: placebo $\left[X^{2}(3)=18.77, p<.001\right]$ y fórmula $\left[X^{2}(3)=22,81, p<.001\right]$.

\section{Discusión}

Los resultados obtenidos tanto en las comparaciones ínter como intra-grupo, permiten avalar la efectividad de fórmula CHB-WAL-WOA en la estimulación del indicador autovaloración de las cualidades personales sobre la actividad de aprendizaje (A), el cual se considera esencial en el mecanismo de reflexión personal.

Tomando en consideración los resultados de las comparaciones intra-grupo, puede afirmarse que la fórmula demostró influir favorablemente en el indicador crítica objetiva acerca del conocimiento obtenido (C), pues dicho grupo mostró cambios significativos en los tres momentos analizados, no ocurriendo así con los que ingirieron el placebo.

En los restantes indicadores, es la metodología empleada la que produce las variaciones positivas. La metodología empleada resulta válida para desarrollar la reflexión personal sobre el aprendizaje de los estudiantes.

\section{Referencias bibliográficas}

Abranches, S. P. (2006). A reflexividade como elemento da prática docente: algums limites para sua efetivação o caso da informática na educação, extraído el 9/5/2005 desde: http://www.educaçãoonline.pro.bra reflexividade como elemento.asp

Alarcão, I (1996). Reflexão crítica sobre o pensamento de D. Schön e os programas de formação de profesores, Revista da Faculdade de Educação, 22(2), 11-42.

Allport, G. W (1965). La personalidad su configuración y desarrollo, Ciudad de La Habana: Edición Revolucionaria.

Bermúdez, R. y Rodríguez, M. (1996). Metodología de la Enseñanza y el Aprendizaje, Ciudad de La Habana: Pueblo y Educación.

Bach, E. (1994). Obras Completas, Ibis, Barcelona.

Coll, C., Palacios, J. y Marchesi, A. (2001). Desarrollo psicológico y educación: Psicología de la educación. Madrid: Alianza.

Coll, C., Palacios, J. y Marchesi, A. (1996). Desenvolvimento Psicológico e Educação Psicología da Educação. Artes Médicas. Porto Alegre Sul. 
(1995). Pensamiento, análisis y autorreg ulación en la actividad cognoscitiva de los alumnos, Ciudad de La Habana: Pueblo y Educación.

Hernández, R.; Fernández, C y Baptista, P. (2006). Metodología de la Investigación (cuarta edición), México DF:.Mc Graw-Hill Interamericana.

Kandel, E.; Schwartz, J. y Jessell, T. (2001). Principios de Neurociencia, Barcelona: Mc Graw-Hill.

Martínez, R. (2001). Modelo de desarrollo de la creatividad pedagógica centrado en la reflexión personal. Santa Clara. ISP «Félix Varela» (Tesis Doctoral)

Rivas, S. (2009). Mecanismos de acción de las esencias florales: una aproximación. En GC-Bach, Cuadernos de Investigación I. (pp. 101-119). Santa Clara: Feijóo.

Rodríguez, B. (2001). Terapia floral y estimulación psicosensorial en el niño con deficiencia psíquica. Barcelona: Índigo.

. (2009). El sistema diagnóstico-terapéutico de Edward Bach: apuntes para una sistematización necesaria. Hereford: Twelve Healers Trust.

Rodríguez, B. y Orozco, R. (2005). Inteligencia emocional y flores de Bach: tipos de personalidad en psicología contemporánea. Barcelona: Índigo.

Semiónov, I. (1983). Enfoque sistemático en el estudio de la organización del pensamiento productivo, en La investigación de los problemas de la Psicología de la actividad creativa. Moscú: Nauta.

Suz, M. (2008). Metodología para el desarrollo de la reflexión personal del profesor en la dirección del proceso docente educativo. Santa Clara: UCLV- ISP «Félix Varela» (Tesis Doctoral) 


\section{Tablas y Gráficos}

Gráfico 1. Comparaciones inter-grupos en los distintos momentos evaluativos
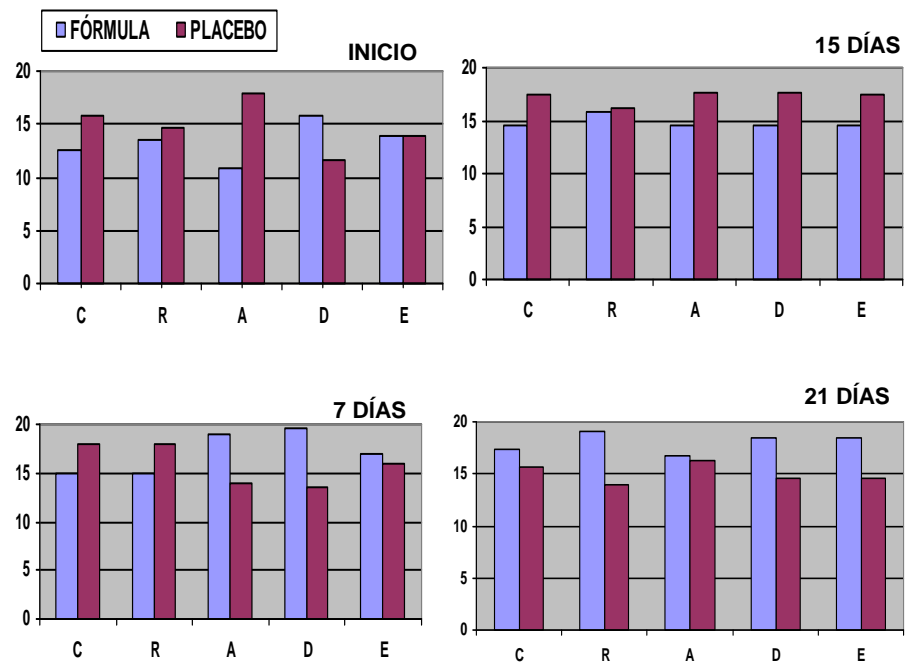

Gráfico 2. Comparaciones intra-grupos en los distintos cortes evaluativos
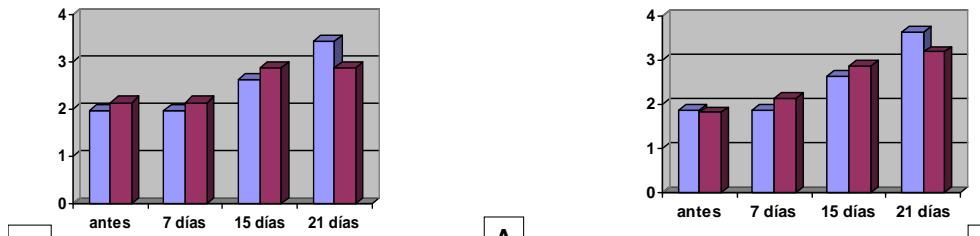

C
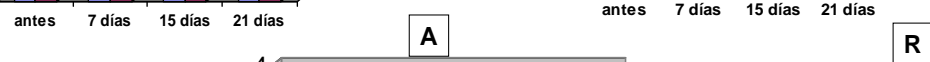

D
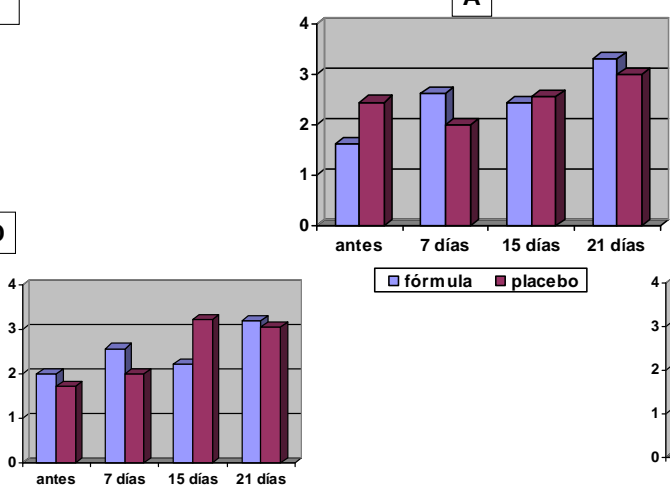

$\square$ fórmula $\square$ placebo

E

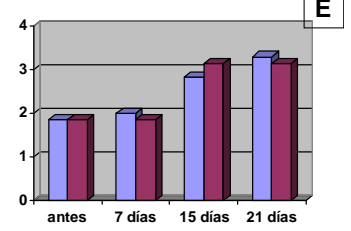




\section{Sobre los autores}

María Suz Pompa. Licenciada en Psicología, Master en Psicopedagogía y Doctora en Ciencias Pedagógicas. Es profesora de la Disciplina de Psicología Educativa de la Faculta de Psicología de la Universidad Central de Las Villas. Su labor como investigadora se ha desarrollado en el área de la Reflexión Personal, tanto de docentes como estudiantes ante el proceso de aprendizaje. Tema que le ha valido premios y reconocimientos, entre los cuales destaca el Premio de la Academia de Ciencias de Cuba. E-mail: suspompa@uclv.edu.cu

Boris C. Rodríguez Martín. Licenciado en Psicología y Master en Psicología Médica. Es profesor de la Disciplina de Psicología General de la Faculta de Psicología de la Universidad Central de Las Villas. Su labor como investigador se ha concentrado en la evaluación de la efectividad de las esencias florales de Bach. Es autor de diversos libros de texto sobre el tema: Esencias florales y estimulación psicosensorial en el niño con deficiencia psíquica (2001); Inteligencia Emocional y Flores de Bach (2005) y El Sistema Diagnóstico-Terapéutico de Edward Bach (2009). Actualmente es el profesor principal del Diplomado en Sistema Diagnóstico Terapéutico de Edward Bach y coordinador del Grupo Científico GC-Bach. 